\title{
In Situ Probing of the Evolution of Irradiation-induced Defects in Copper
}

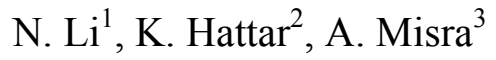 \\ 1. Los Alamos National Laboratory, Los Alamos, NM 87545, USA \\ 2. Sandia National Laboratories, Albuquerque, NM 87185, USA \\ 3. University of Michigan, Ann Arbor, MI 48109, USA
}

Any attempt to produce nuclear materials with improved mechanical properties depends heavily on understanding the root causes of radiation damage, which are individual and clustered vacancies and interstitials (self or external) produced during collision cascades between energetic particles and target atoms [1,2]. The subsequent diffusion and clustering of these defects, along with the associated transport of impurities, will dramatically change the microstructure and lead to accelerated degradation of properties during irradiation. In order to effectively suppress irradiation-induced damage, significant research is being conducted to synthesize materials containing stable sinks for trapping and recombining irradiation-induced point defects [3,4]. However, experimental evidence through in situ studies is seldom provided [5] due to both the tediousness of identifying boundary types and the difficulty of directly performing experiments observing the interaction of boundaries with those defects.

In order to unravel the evolution of irradiation damage with the dose, we performed in situ ion irradiation experiments in a transmission electron microscope (TEM). The present study will focus on a common grain boundary in $\mathrm{Cu}$, the $\Sigma 3\{112\} \|\{112\}$ incoherent twin boundary (ITB). Through in situ $\mathrm{Cu}^{3+}$ ion irradiation at room temperature in a TEM, we have investigated the evolution of defect clusters as a function of the radiation dose at different distances from the $\Sigma 3\{112\}$ ITB in $\mathrm{Cu}$. During irradiation, defect clusters evolve through 4 stages: (i) incubation, (ii) non-interaction, (iii) interaction and (iv) saturation; and the corresponding density was observed to initially increase with irradiation dose and then approach saturation (in Fig. 1). No denuded zone is observed along the $\Sigma 3\{112\}$ ITB and the configuration of defects at the boundary displays as truncated SFTs (in Fig. 2). Several defect evolution models have been proposed to explain the observed phenomena [6].

In summary, current study emphasis will be placed on the defect evolution as a function of the radiation dose at different distance to the boundary. These results will provide a useful basis for analyzing the influence of interface sink strength on the reduction of radiation-induced defects [7].

[1] M.J. Demkowicz, P. Bellon, and B.D. Wirth, MRS Bull. 35 (2010), p. 992.

[2] B.D. Wirth, Science 318 (2007), p. 923.

[3] T. Allen et al, MRS Bull. 34 (2009), p. 20.

[4] Y. Guerin, G.S. Was, and S.J. Zinkle, MRS Bull. 34 (2009), p. 10.

[5] A.H. King and D.A. Smith, Philos. Mag. A 42 (1980), p. 495.

[6] N. Li, K. Hattar, and A. Misra, Journal of Nuclear Materials, 439 (2013), p. 185.

[7] This work is supported by the Center for Materials at Irradiation and Mechanical Extremes

(CMIME), an Energy Frontier Research Center (EFRC) under Award No. 2008LANL1026 by the DOE, Office of Science, Office of Basic Energy Sciences. 

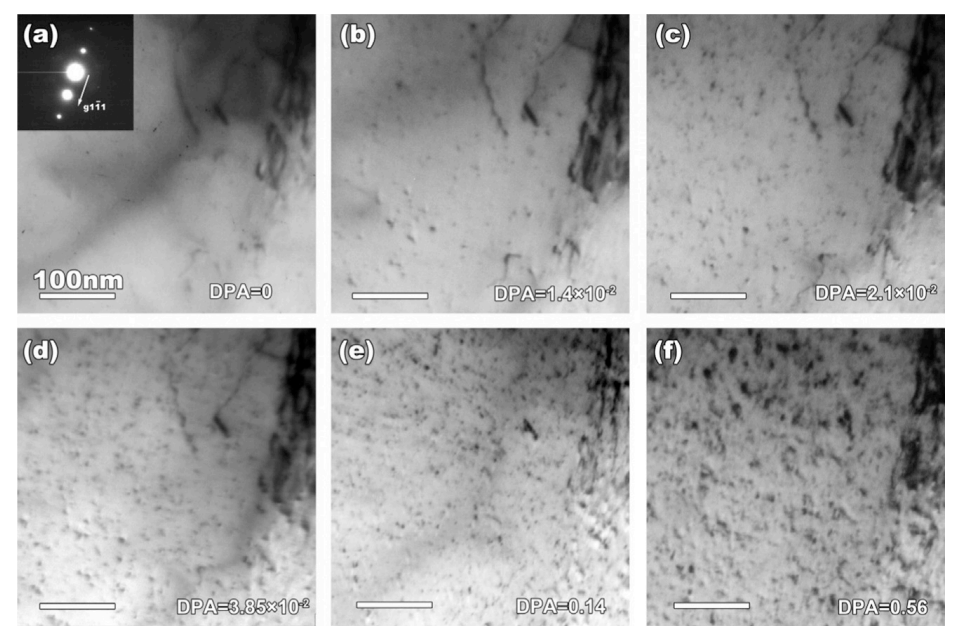

Figure 1. (a) Cross-sectional TEM Cu foils tilted to two-beam conditions with $\mathrm{Cu} 1 \overline{1} 1$ spots strongly excited. $(b-f)$ A sequence of TEM video images recording the microstructure evolution of pure $\mathrm{Cu}$ under $3 \mathrm{MeV} \mathrm{Cu}^{3+}$ irradiation at room temperature.
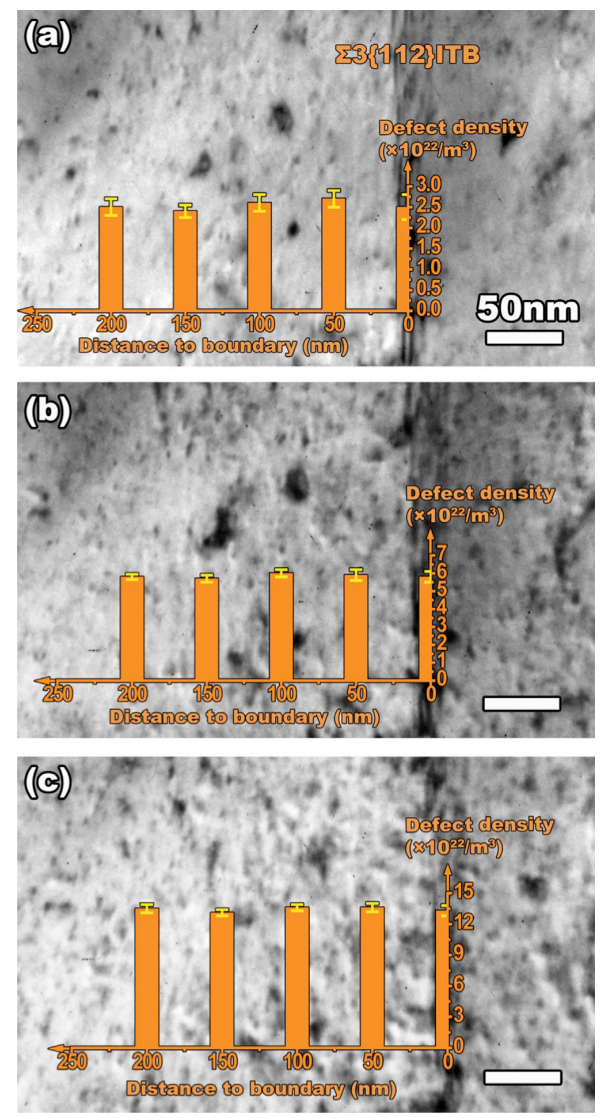

Figure 2. $(\mathrm{a}-\mathrm{c})$ The distribution of radiation-induced defects as a function of the distance from the boundary at three different doses. 